\title{
BMJ Open Biomarkers of autonomic regulation for predicting psychological distress and functional recovery following road traffic injuries: protocol for a prospective cohort study
}

Ilaria Pozzato, ${ }^{\circ 1}$ Ashley Craig, ${ }^{\circ 2}$ Bamini Gopinath, ${ }^{1,3}$ Yvonne Tran, ${ }^{1}$ Michael Dinh, ${ }^{4}$ Mark Gillett, ${ }^{5}$ Ian Cameron ${ }^{1}$

To cite: Pozzato I, Craig A, Gopinath B, et al. Biomarkers of autonomic regulation for predicting psychological distress and functional recovery following road traffic injuries: protocol for a prospective cohort study. BMJ Open 2019;9:e024391. doi:10.1136/ bmjopen-2018-024391

- Prepublication history for this paper is available online. To view these files, please visit the journal online (http://dx.doi. org/10.1136/bmjopen-2018024391).

Received 24 May 2018

Revised 14 January 2019

Accepted 4 March 2019
Check for updates

(C) Author(s) (or their employer(s)) 2019. Re-use permitted under CC BY-NC. No commercial re-use. See rights and permissions. Published by BMJ.

For numbered affiliations see end of article.

Correspondence to Professor Ashley Craig; a.craig@sydney.edu.au

\section{ABSTRACT}

Introduction Psychological distress is a prevalent condition often overlooked following a motor vehicle crash (MVC), particularly when injuries are not severe. The aim of this study is to examine whether biomarkers of autonomic regulation alone or in combination with other factors assessed shortly after MVC could predict risk of elevated psychological distress and poor functional recovery in the long term, and clarify links between mental and physical health consequences of traffic injury.

Methods and analysis This is a controlled longitudinal cohort study, with follow-up occurring at 3,6 and 12 months. Participants include up to 120 mild to moderately injured MVC survivors who consecutively present to the emergency departments of two hospitals in Sydney and who agree to participate, and a group of up to 120 nonMVC controls, recruited with matched demographic characteristics, for comparison. WHO International Classification of Functioning is used as the framework for study assessment. The primary outcomes are the development of psychological distress (depressive mood and anxiety, post-traumatic stress symptoms, driving phobia, adjustment disorder) and biomarkers of autonomic regulation. Secondary outcomes include indicators of physical health (presence of pain/fatigue, physical functioning) and functional recovery (quality of life, return to function, participation) as well as measures of emotional and cognitive functioning. For each outcome, risk will be described by the frequency of occurrence over the 12 months, and pathways determined via latent class mixture growth modelling. Regression models will be used to identify best predictors/biomarkers and to study associations between mental and physical health.

Ethics and dissemination Ethical approvals were obtained from the Sydney Local Health District and the research sites Ethics Committees. Study findings will be disseminated to health professionals, related policy makers and the community through peerreviewed journals, conference presentations and health forums.

Trial registration number ACTRN12616001445460.
Strengths and limitations of this study

- This study will comprehensively describe the risk of developing psychological distress and poor functional recovery in people involved in a motor vehicle crash and sustaining mild-to-moderate injuries.

- Identifying biomarkers of mental health risk and poor functional recovery adds novelty to this field. Easy-to-measure biomarkers of autonomic regulation hold promise in preventing serious mental disorders, reducing patient-related barriers to mental healthcare and improving recovery rates among crash survivors.

- The use of the International Classification of Functioning standard framework and validated measures will assure an in-depth analysis of complex needs of the injured person, which are often studied in isolation, and will promote comparability with other research.

- Findings may be useful to primary care clinicians in the early diagnosis and referral of at-risk individuals for a better management of mental health and a more integrated form of care.

- Findings may also assist changes in compensation processes and policies towards a more holistic approach to injury recovery.

\section{INTRODUCTION}

Around 50 million people worldwide are injured annually in non-fatal motor vehicle crashes (MVC). ${ }^{1}$ Surviving a crash can lead to a number of physical and psychological consequences, and many individuals develop longterm disability. ${ }^{2-4}$ While improved outcomes are shown for severe injuries, ${ }^{5}$ evidence suggests that minor-to-moderate injuries are a challenge ${ }^{67}$ With traffic injuries remaining the 10th leading cause of disability-adjusted life years in $2010,{ }^{8}$ minor-to-moderate injuries (ie, Maximum Abbreviated Injury Score [MAIS] of less than 3) account for $46 \%$ of 
injury-related disability. ${ }^{3}$ Therefore, consequences and costs of minor-to-moderate traffic injuries such as whiplash, mild traumatic brain injury and musculoskeletal injury are serious public health concerns. ${ }^{6}$

A recent longitudinal study from Europe reported that minor-to-moderate (MAIS $<3$ ) traffic injuries hospitalised for more than 24 hours result in higher average costs, both direct and indirect, compared with severe injuries. ${ }^{9}$ On the other hand, in Australia, it is estimated that around US\$1b is spent each year only on traffic injuries admitted to hospital for less than 24 hours, with an average cost by casualty of US $\$ 14183 .{ }^{10}$ Furthermore, when psychological problems are involved, care costs have been shown to escalate alarmingly and time to recovery to increase fourfold. ${ }^{11}$

Psychological distress is prevalent in people surviving an MVC $^{11-16}$ and has been demonstrated to be a substantial contributor to long-lasting morbidity and disability, ${ }^{7} 1718$ affecting up to one in two MVC survivors, according to a recent meta-analysis. ${ }^{19}$ Although there is some variability in estimates, the proportion of MVC survivors (up to $50 \%$ ) who experience psychological problems appears to be many times greater than the proportion of people with mental health in the Australian community $(20 \%)^{20}$ and global population $(25 \%),{ }^{21}$ often resulting in serious mental disorders, such as post-traumatic stress disorder (PTSD), major depressive disorder, driving phobias and other anxiety disorders. ${ }^{13} 14$

Unlike physical consequences that ideally receive appropriate and timely medical care following an MVC, early clinical management of psychological consequences associated with mild-to-moderate physical injury rarely occurs since, for example, psychological distress is often normalised as a common response to the trauma. Hence, it is not surprising that mental health-related quality of life of MVC survivors is more resistant to improvement over time than physical-related outcomes, as for instance demonstrated by Kenardy et al. ${ }^{17}$ This is pertinent given failure to address psychological distress will have a significant detrimental impact on functional recovery, ${ }^{22}$ supporting the hypothesis that there is 'no health without mental health'. ${ }^{21}$

Some underlying circumstances may lead to delayed recognition of psychological distress. First, a clear diagnostic framework is lacking for detecting psychological distress symptoms that include clinically elevated anxiety and depressive mood and high levels of arousal, ${ }^{19}$ and which often co-occur following an MVC. ${ }^{14}$ It is argued that these symptoms may be all manifestations of one construct of general distress. ${ }^{23}$ However, previous studies on MVC survivors typically focused on a single mental disorder, particularly PTSD. The second and more significant fact is the absence of empirical evidence for reliable biomarkers of vulnerability to psychological distress following an MVC.

Vulnerability to psychological distress and poor functional recovery draws on the broader concept of psychological resilience as the unique ability of an individual to adapt to adversity, which develops throughout the lifespan. ${ }^{24}$ Following an MVC, individual vulnerability to poor psychological adjustment and functional recovery, has been linked to a number of biological (eg, preinjury health, injury severity), psychological (eg, prior traumatic experiences, perceptions) and environmental factors (eg, social support, compensation) ${ }^{422} 25$; however, biological responses, in particular autonomic responses, to traffic injury remain an underinvestigated area.

Unlike other traumatic or pathological conditions, there have been very few studies examining the predictive value of biomarkers of autonomic regulation, such as traditional vital signs ${ }^{26-31}$ measured early after an MVC, to investigate long-term outcomes, and only one study, to our knowledge, has attempted to use heart rate variability (HRV) ${ }^{32}$ The main focus of these studies was the association between heart rate at the scene or during hospitalisation and the development of PTSD following an MVC. However, there was lack of consistency between findings, highlighting differences in methodology and confirming that substantial interindividual variability exists in psychobiological responses to physical threat.

Biological responses to potential threats involve activation of neural substrates such as the stress response system. The autonomic nervous system is the first to respond to stressors by a transitory withdrawal of the parasympathetic 'brake' and modulation of sympathetic activity for a rapid control of body functions critical for survival, that is, changes in heart rate, blood pressure and breathing rate, and later re-engagement of the parasympathetic system during recovery, by inhibition of sympathetic control.

Studying the sympathetic-parasympathetic balance is rather complex, and one marker is often not enough to convey the complexity of stress regulatory processes. ${ }^{33}$ Therefore, more research is needed to assess autonomic resting level, reactivity and recovery ${ }^{34}$ in a comprehensive manner, by using several markers to increase predictive ability ${ }^{24} 35$ and investigating their relationship with other psychological, social and biological factors. There is emerging evidence of associations between negative affect, perseverative cognition and physiological over-reactivity to stress, ${ }^{24}{ }^{34}$ as introduced, for example, by the perseverative cognition hypothesis. ${ }^{36}$ The most common autonomic markers include vital signs (ie, heart rate, respiratory rate, blood pressure, peripheral skin temperature), skin conductance (SC), peripheral blood circulation and HRV. While SC and peripheral skin temperature have been widely used as an index of sympathetic activity, $\mathrm{HRV}$, the variation in the beat-to-beat interval, is recommended as a reliable, non-invasive index for assessing cardiac vagal tone. Higher levels of vagally mediated resting-state $\mathrm{HRV}^{34}$ and faster cardiovascular recovery ${ }^{24}$ have been shown to be associated with better self-regulatory capacity to environmental demands.

Different theoretical models ${ }^{37-39}$ have linked vagally mediated HRV to individual differences in adaptation to external and internal stressors, suggesting that poor 
autonomic regulation is an indicator of poor self-regulatory capacity in managing stress levels, that can be associated with poor psychological health, ${ }^{40}{ }^{41}$ physical health, ${ }^{4243}$ cognitive performance ${ }^{38} 43$ and social functioning. ${ }^{37} 41$ Thus, sympathetic-parasympathetic balance, measured early after an MVC, is regarded as a promising biomarker of vulnerability to develop dysfunctional responses, such as elevated psychological distress and stress-related disorders that can affect a person's capacity to recover following traffic injury. ${ }^{44}$

There is substantial evidence that supports the co-occurrence of psychological and physical consequences post-MVC, for example, anxiety, depression, chronic pain, fatigue, sleep disturbance and other somatic conditions, ${ }^{25} 45$ for which physical evidence is often missing, hinting at the possibility of shared psychological and biological factors in stress vulnerability. ${ }^{46-48}$ However, the majority of evidence for these associations comes from cross-sectional studies, especially from the relationship between chronic pain and PTSD in whiplash injuries. Therefore, prospective research is now needed to investigate links between mental and physical health in recovery from traffic injuries.

Full functional recovery is a combination of a person's physical and mental well-being, often measured as health-related quality of life, and social functioning, such as resumption of work/usual level of activities and social participation. ${ }^{49}$ In the current study, WHO International Classification of Functioning (ICF ${ }^{50}$ will be employed as the biopsychosocial framework to assess mental health and functional recovery outcomes following MVC-related injury, and to build comprehensive prognostic models for the identification of the at-risk cohort. Early detection is crucial for preventing disease and progression to chronicity; therefore, this study has the potential to prevent the development of serious mental health conditions and disability among thousands of MVC survivors in Australia and worldwide annually.

\section{Study aims}

The study objectives are:

1. To determine prevalence estimates and trajectories of psychological distress (ie, depressive mood and anxiety, PTSD symptoms, driving phobia, adjustment disorder) and functional recovery (ie, health-related quality of life, return to work/activities, social participation) post-MVC.

2. To determine the efficacy of autonomic biomarkers (ie, post-MVC vital signs and autonomic measures 1-month post injury), alone or in combination with other biopsychosocial factors, in predicting psychological distress and poor functional recovery post MVC.

3. To investigate associations and shared biopsychosocial predictors/biomarkers, between mental health (ie, psychological distress, emotional and cognitive functioning) and physical health (ie, pain, fatigue, physical functioning) consequences post MVC.

\section{METHODS AND ANALYSIS \\ Conceptual framework}

The conceptual study framework, drawing on WHO ICF model ${ }^{50}$ is outlined in figure 1 . WHO ICF Disability and Health model is widely recognised as the most recent and comprehensive framework for assessing health and disability. Therefore, it provides an effective framework for describing consequences of road traffic injuries, such as psychological distress and poor functional recovery.

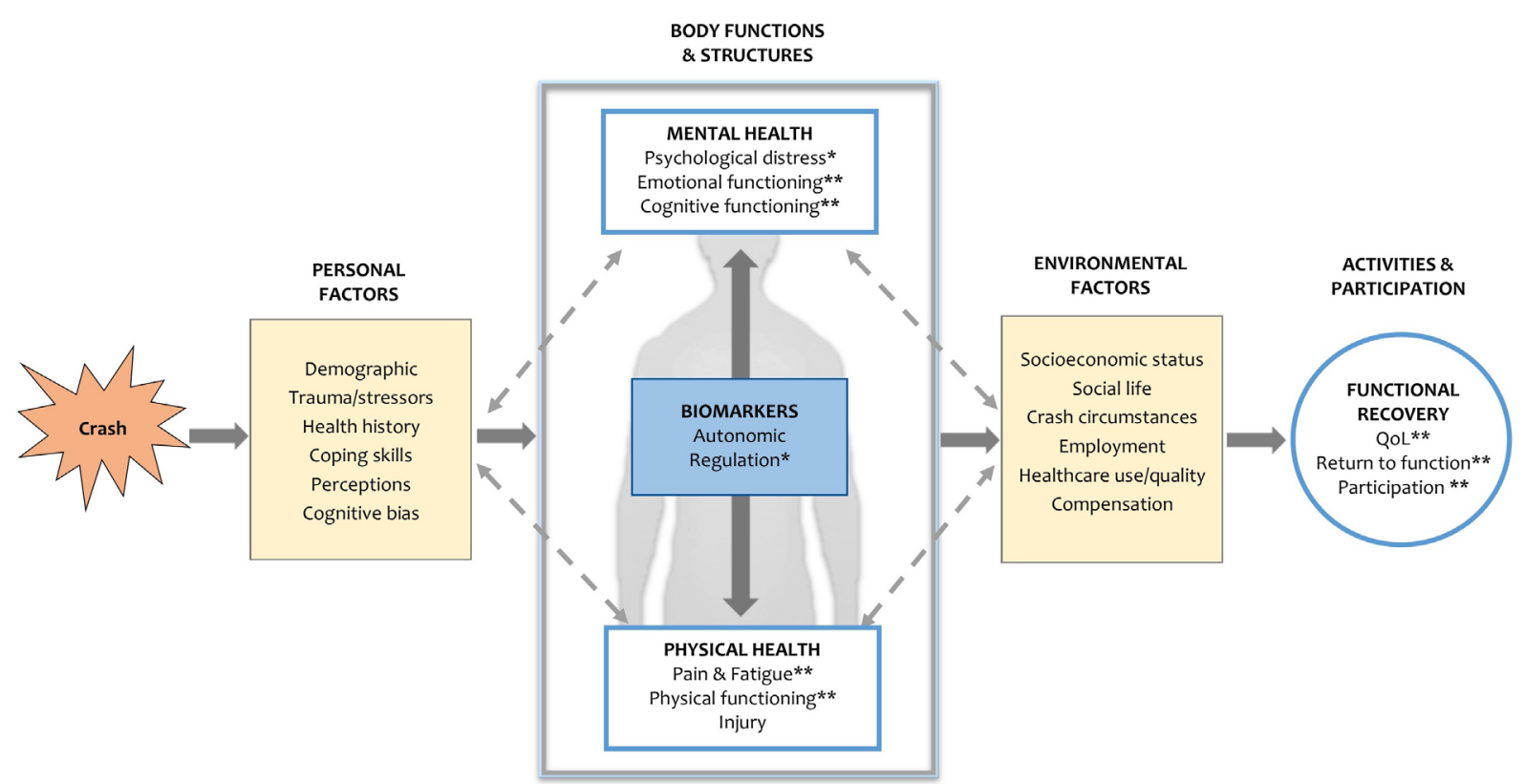

Figure 1 International Classification of Functioning-based on the impact of psychological distress on recovery from injuries sustained in a traffic crash (IMPRINT) study biopsychosocial assessment framework. *Primary outcomes; ${ }^{\star *}$ secondary outcomes. QoL, quality of life. 
Potential predictors will be biological, personal and environmental factors, with a focus on testing the predictive ability of autonomic biomarkers, which are simple and easy to measure. This is a prognostic study where event prediction will follow a data-driven approach and careful consideration will be given to the issue of overfitting. ${ }^{51}$ There are also plans of exploring causal effects that will come as a separate proposal.

\section{Design}

A controlled longitudinal cohort design will be undertaken to assess risk of psychological distress and poor functional recovery among mildly to moderately injured MVC survivors (Injury Severity Score ${ }^{52} \leq 15$ ) ${ }^{53-55}$ Baseline assessment will occur around 1 month (3-6 weeks) of their injury, with follow-up assessments occurring at 3, 6 and 12 months post injury. Results will be compared with a control group who have not sustained an MVC or severe injuries in the past 5 years, and who will be assessed at the same time periods.

\section{Setting}

The emergency departments (ED) of two of the seven adult major trauma services in NSW, Australia, will be involved in the recruitment of MVC survivors: Royal North Shore Hospital and Royal Prince of Alfred Hospital. ${ }^{56}$ These are large public hospitals located in Sydney metropolitan area, providing care to trauma patients across the whole spectrum of severity.

\section{Participants' eligibility and identification}

The study inclusion and exclusion criteria for MVC survivors and control participants are presented in table 1 . These include exposure status (ie, injury vs non-injury) and factors related to the outcomes being studied (ie, mental health, autonomic function, disability and physical health). At the time of their enrolment, both cohorts will be free of severe mental health disorders or major disabling conditions due to very severe injury like spinal cord injury. The non-injured cohort will include normally healthy individuals with no active medical concern or serious comorbid conditions (ie, chronic disease, degenerative neurological disease). Medications or other factors that could potentially influence autonomic function/ outcomes investigated, as well as differences between the two cohorts, will be adjusted for in the analysis.

The identification of $M V C$ survivors will begin following their presentation to the ED of the two hospitals. Participants will be consecutively identified and assessed for eligibility by a research nurse from the information management system, FirstNet.

The non-MVC control group, matched by sex and age $( \pm 5$ years) to the MVC sample on a case-by-case basis, will be recruited through advertising. Study eligibility will be assessed using an online self-reported screening interview.

\section{Recruitment process}

Participants' recruitment flow chart is illustrated in figure 2. Eligible survivors will be invited to participate by: (1) being approached at the time of ED presentation, or (2) receiving an invitation letter to their postal address from the ED-treating clinician. After written consent is obtained (opt-in approach), participants will be requested to complete the baseline interview-based assessment by accessing an online secure website, and otherwise by completing a paper based or phone interview. As part of their enrolment, within 6 weeks of their

Table 1 Inclusion and exclusion criteria of motor vehicle crashes (MVC) participants and non-MVC controls

\begin{tabular}{|c|c|c|}
\hline & MVC participants & Non-MVC controls \\
\hline $\begin{array}{l}\text { Inclusion } \\
\text { criteria }\end{array}$ & $\begin{array}{l}\text { Age } \geq 18 \text { years. } \\
\text { Sustained in last } 6 \text { weeks, a minor-to-moderate injury } \\
\text { due to MVC in NSW, Australia. } \\
\text { A driver, motorbike rider, passenger, pillion passenger, } \\
\text { pedestrian and bicyclist (only collision involving a } \\
\text { motorised vehicle). } \\
\text { Presented to RNSH or RPAH emergency departments, } \\
\text { Sydney, Australia. } \\
\text { Sufficient English proficiency. }\end{array}$ & $\begin{array}{l}\text { Age } \geq 18 \text { years. } \\
\text { No history of MVC, serious injury or traumatic } \\
\text { event (ie, w/perceived threat of injury to self or } \\
\text { others) in the previous } 5 \text { years. } \\
\text { Sufficient English proficiency. }\end{array}$ \\
\hline $\begin{array}{l}\text { Exclusion } \\
\text { criteria }\end{array}$ & $\begin{array}{l}\text { Catastrophic injury as defined by NSW are lifetime care, } \\
\text { that is, a very severe traumatic brain injury, a spinal } \\
\text { cord injury, extensive burns to the body (>60\%), major } \\
\text { amputation or blindness. } \\
\left.\text { Serious injury (ISS }{ }^{52}>15\right) .53 \\
\text { Localised, superficial soft-tissue injuries. } \\
\text { MVC due to intentional self-harm or MVC survivors with } \\
\text { severe mental health condition. } \\
\text { Death of a family member in the MVC. } \\
\text { Dementia or cognitive impairment affecting ability to } \\
\text { consent. }\end{array}$ & $\begin{array}{l}\text { Catastrophic injury: severe brain injury, spinal } \\
\text { cord injury, extensive burns to the body, major } \\
\text { amputation or blindness. } \\
\text { Severe mental health condition. } \\
\text { Dementia or cognitive impairment affecting ability } \\
\text { to consent. } \\
\text { Chronic disease such as severe heart disease, } \\
\text { stroke, cancer, chronic respiratory diseases, } \\
\text { obesity and advanced complicated diabetes. } \\
\text { Degenerative neurological disease. }\end{array}$ \\
\hline
\end{tabular}

ISS, Injury Severity Score; RNSH, Royal North Shore Hospital; RPAH, Royal Prince of Alfred Hospital. 


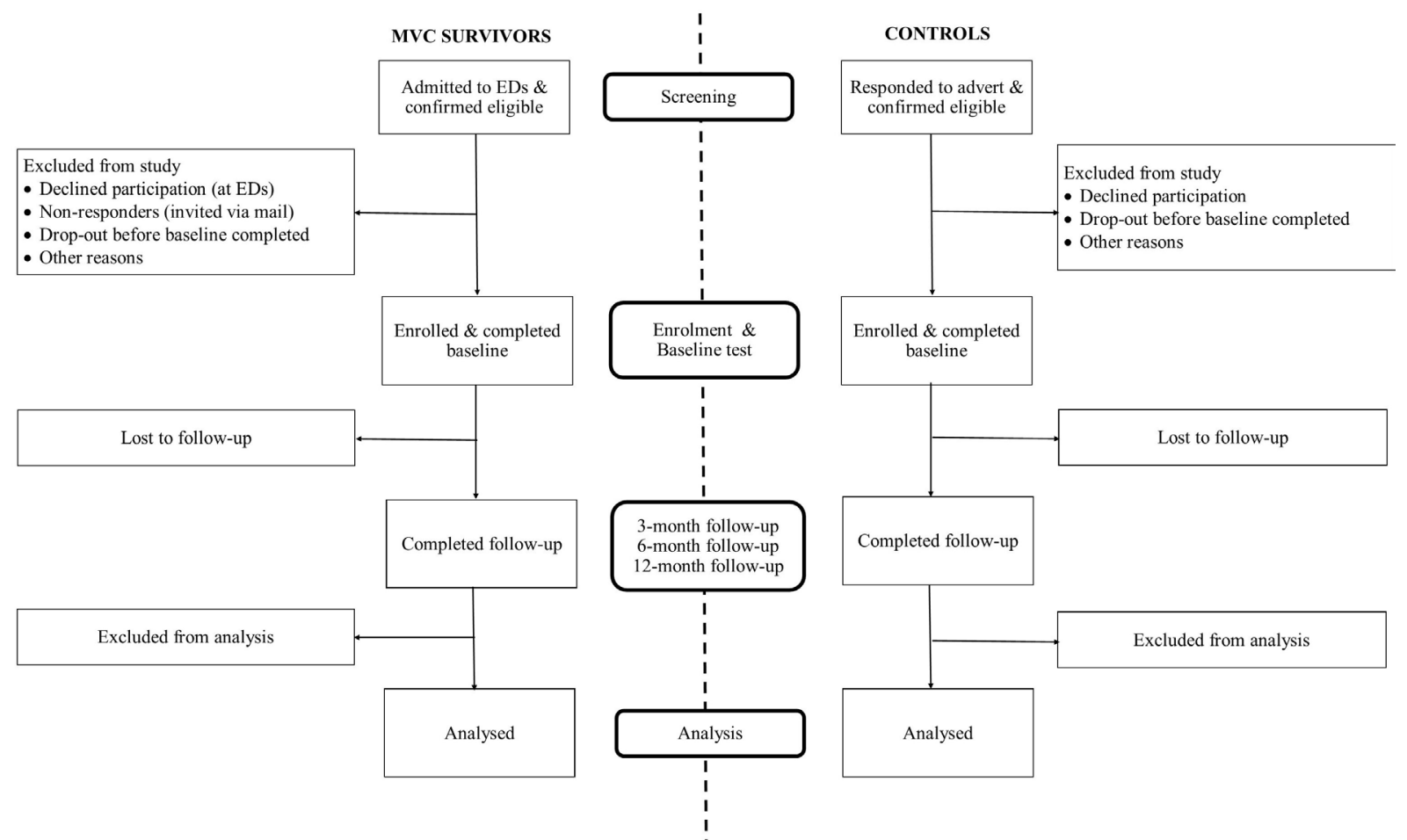

Figure 2 IMPRINT study participants recruitment flow chart. ED, emergency department; MVC, motor vehicle crashes.

accident, participants will also be asked to attend a $30 \mathrm{~min}$ hospital visit for the collection of autonomic biomarkers. An overall reimbursement up to US\$100 in vouchers will be provided to cover participant travel cost and time.

\section{Sample size}

A sample size of up to 120 MVC participants and an equivalent number of non-MVC controls will be employed to test the study aims. Assuming a loss to follow-up of $20 \%-30 \%$, a moderate effect size of 0.2 (eta-squared or $\eta^{2}$ ) at $\alpha=0.05$, two groups and four time periods, statistical power using repeated measures analyses was calculated to be $83 \% .^{57}$ For the logistic regression, assuming an $\alpha=0.05$, 10 predictors, a moderate effect size of 0.2 (eta-squared or $\eta^{2}$ ), the power to measure the strength of relationship was calculated to be $90 \% .^{57}$

\section{Assessments and outcome measures}

Assessment time points, study outcomes and details of the validated measures used within each ICF domain are provided in table 2 .

\section{Primary outcomes}

The primary outcome is the development of psychological distress as any psychological discomfort that interferes with daily living functioning following the MVC. Psychological distress is defined as the presence of at least one of the following psychological symptoms over the 12 months after the MVC, including elevated depressive mood and anxiety (Depression, Anxiety and Stress Scale) ${ }^{58}$ and PTSD symptoms (Impact of Events Scale) ${ }^{59}$ evaluated using these two validated psychometric assessments, as well as the presence of driving phobia and adjustment disorder using diagnostic interview (Mini-International Neuropsychiatric Interview based on Diagnostic and Statistical Manual of Mental Disorders, 5th Edition). ${ }^{60}$ Another key outcome are biomarkers of autonomic regulation by means of HRV and other autonomic measures of sympathetic-parasympathetic balance as described below.

\section{Secondary outcomes}

Secondary outcomes include indicators of physical health (ie, pain, fatigue and physical functioning measures such as somatic symptoms and/or new clinical diagnosis) and functional recovery (ie, health-related quality of life, return to work/usual activities, social participation) up 12 months post MVC. Other outcomes of interest are measures of emotional (ie, emotional balance, self-reported valence and arousal of affect) and cognitive functioning (ie, perceived cognitive health, selective attention and processing speed ability).

\section{Predictors}

Biopsychosocial predictors include a comprehensive regimen of biological (eg, injury details), personal (eg, demographic, health history, perceptions/reaction to the injury/expectations, coping skills, self-efficacy, cognitive styles) and environmental factors (eg, crash circumstances, economic factors, social life, compensation, healthcare use and satisfaction) that may predict risk of elevated psychological distress and poor recovery, in association with autonomic biomarkers.

\section{Biomarkers of autonomic regulation}

Measures and procedures

Biomarkers of autonomic regulation consist of vital signs (heart rate, respiratory rate, blood pressure) on the day 


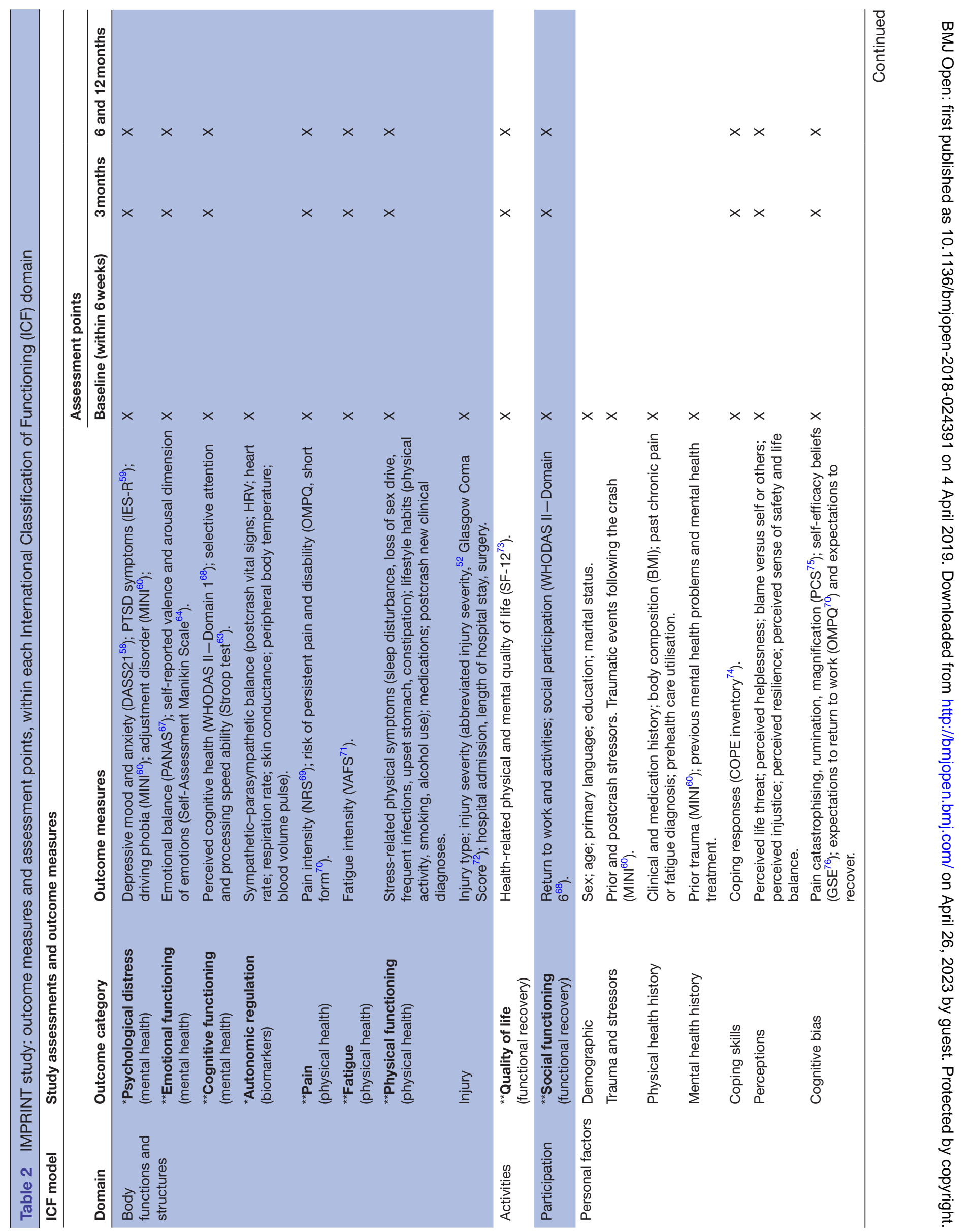


of the accident (at the scene and ED triage) ${ }^{26}$ and autonomic measures around 1 month (3-6 weeks) of the injury, non-invasively and simultaneously acquired using a BiosemiTM Active-Two System at a $2480-\mathrm{Hz}$ native sampling rate. Autonomic assessment will include respiration rate (sensitive band around chest), SC level and responsiveness, that is, absolute $S C$ and number/amplitude of non-specific SC fluctuations (surface electrodes on second and fourth fingers of non-dominant hand), peripheral body temperature (clip-on sensor on fifth finger of non-dominant hand), blood volume pulse amplitude (light sensor on third finger of non-dominant hand), heart rate and $H R V$ (ECG recording). ${ }^{61}$ For the ECG recording, three Ag/ $\mathrm{AgCl}$ electrodes will be placed in modified Einthoven I and II configurations (beneath the right and left clavicles, and on the fourth intercostal space on the left side of the sternum).

\section{Experimental design and structure}

The chosen design allows examination of within and between subject changes between phases. For an in-depth investigation of autonomic regulatory processes, a $3 \mathrm{R}$ protocol $^{34}$ will be employed that allows examination of tonic resting level (baseline) and phasic autonomic control, including autonomic reactivity (change between baseline and task) and recovery (change between task and post-task, percentage/time of return to baseline).

On the morning prior to the autonomic assessment, participants will be asked to follow a normal sleep routine and to abstain from eating, drinking caffeinated and alcoholic beverages, smoking and intense physical activity. Recording will be conducted under constant conditions, between 08:00 and 13:00 to control circadian influences. ${ }^{62}$ Participants will sit in a relaxed position with eyes open. The protocol includes four phases, throughout which autonomic biomarkers will be measured continuously: (1) 5 min resting baseline, after a $2 \mathrm{~min}$ acclimatisation; (2) 5 min response inhibition task, using the validated Stroop Color-Word test ${ }^{63}$; (3) 5 min resting post-task; (4) 2 min slow-paced breathing (6 breaths/min), using a breath pacer application providing visual feedback for breathing cycle control. Before every phase change, participants will be asked to rate pain and fatigue intensity, and their emotional state (Self-Assessment Manikin Scale). ${ }^{64}$

\section{Data manipulation}

After appropriate data cleaning to remove noise and artefacts and resampling (512-Hz sampling rate), biosignals analysis will be performed using Kubios (V.2.0 beta 2, Department of Applied Physics, University of Kuopio, Finland ${ }^{65}$ and other Analysis Software. For HRV, time-domain, frequency-domain (LF: $0.04-0.15 \mathrm{~Hz}$; HF: $0.15-$ $0.4 \mathrm{~Hz}$ ) and non-linear indices will be analysed.

\section{Hypotheses and data analysis}

It is hypothesised that:

a. People sustaining mild-to-moderate injuries in an MVC will continue to suffer high rates of psychological 
distress, poor physical health (eg, high levels of pain and fatigue, poor physical functioning) and poor functional recovery (eg, poor quality of life, limited work/ activities resumption and low social participation) 6-12 months post injury, though improvements in all outcomes are expected to occur over time compared with 1-3 months post injury.

b. Compared with non-MVC controls, MCV survivors will have significantly poorer autonomic regulation (ie, lower sympathetic-parasympathetic balance) at 1 month follow-up, and will exhibit poorer mental health (eg, higher psychological distress, poorer emotional and cognitive functioning), poorer physical health (eg, higher levels of pain and fatigue, lower physical functioning) and poorer social outcomes (eg, poorer health-related quality of life and social functioning) over the follow-up period, though to a lesser extent at long term (6 and 12 months) compared with short term (1-3 months) post injury;

c. Among MVC survivors, those with sustained elevated psychological distress over the first-year post injury will be less likely to recover and have significantly lower autonomic regulation early after the crash (ie, higher heart rate and respiratory rate on the day of the accident; poorer sympathetic-parasympathetic balance 1 month post injury) compared with those without psychological distress who will be more likely to recover;

d. Specific biological factors (eg, higher injury severity, longer hospital stay), personal factors (eg, older age, being female, poor preinjury health, poor coping skills, negative perceptions/reactions to the injury, catastrophic thinking, low self-efficacy, poor recovery expectations) and environmental factors (eg, preinjury unemployment, poor socioeconomic background, protected road users, poor social support, poor healthcare use and satisfaction, involvement in compensation) will predict increased risk of elevated psychological distress and poorer functional recovery in those injured in an MVC.

e. There will be similarities in factors predicting mental and physical health consequences of a traffic injury. Shared predictors/biomarkers will include biomarkers of poor autonomic regulation, and psychological/ personal factors like preinjury health, psychological distress, thinking styles, coping skills and self-efficacy.

f. In both groups, there will be high correlations between states of low sympathetic-parasympathetic balance, reactivity to stress and negative emotions/perceptions. Those with balanced autonomic regulation will be more likely to exhibit better health (ie, both mental and physical health) and social outcomes (quality of life, social functioning).

All data analyses will be performed using SPSS software V.22. Descriptive statistics will define sociodemographic characteristics and other variables of interest of the study. First, risk will be calculated separately for each outcome by the frequency of occurrence and percentage over the 12-month post-MVC period, while latent class mixture growth modelling will determine outcome class pathways. In addition, repeated measures linear mixed models will be used to analyse differences between and within groups over time.

To identify biomarkers/predictors at 3, 6 and 12 months post MVC, multiple regression analyses will be conducted for each outcome of interest, after appropriate selection procedures (eg, univariate analysis, bootstrap), whereas adjustment for confounders will not be considered as priority given the non-aetiologic nature of the study. ${ }^{51}$ Sensitivity/specificity analyses and constructing receiver operating characteristic plots will determine discriminative performance.

Subgroup analyses and multiple regression will study differences and shared predictors/biomarkers between mental and physical health outcomes following the MVC. Clinical significance of autonomic biomarkers will be also tested using cluster analysis and other exploratory analyses.

The same analyses will be conducted with control data, to describe differences in outcomes with the group of MVC survivors.

\section{Bias}

To reduce selective bias, the study will adopt the following strategies: a 6-week inception period, participant cost reimbursement, adequate statistical power, as well as consecutive recruitment from two hospitals with major catchments for traumatic MVC injury in Sydney that will provide a wide variety across ethnicity, culture, socioeconomic status and education levels.

To minimise loss to follow-up, multiple options will be given on assessment modality (online, paper based on phone interview) and date/time to schedule the hospital visit. Follow-up reminders via emails and phone calls will also increase adherence through the study.

Though experienced research staff will ensure quality and completeness of assessment, missing data are expected to occur. Imputation techniques will be used for missing values, considering comparison with non-missing cases. The study will also aim to assess a wide range of possible prognostic markers on their ability to identify people with poor outcomes, for ease of use when translating the research findings to clinical practice. $^{51}$

\section{Patient and public involvement}

Research question and outcome measures are developed with knowledge from our ongoing research into patient feedback, expectations and concerns following traumatic injury. However, the present study is not a clinical trial; therefore, participants are not directly involved in study design, conduct or assessment of intervention. Nevertheless, findings from this study, that is, peer-reviewed publications, will be shared with study participants via email communication. 


\section{ETHICS AND DISSEMINATION}

\section{Approvals, progress and timeframe}

STROBE guidelines have been followed for this protocol.

The first participant was recruited on 16 June 2017, and both sites are actively contributing towards the recruitment target. It is anticipated that the recruitment will last until the end of March 2019; thus, data collection is expected to be completed by early 2020 .

\section{Data storage and sharing}

Eligible participants' information will be stored on the local health server at the site, until informed consent is obtained. Signed consent forms will be stored in lockable cabinets. Data from consenting participants will be then entered and stored on a secure online platform (REDCap) at The University of Sydney, to which only designated research team will have access. Methodology details and deidentified data will be available on request once analyses have been completed, provided there is no breach of participant confidentiality.

\section{Confidentiality and safety aspects}

Written informed consent will be sought from participants and for publication of deidentified study findings. Participants will be reminded that their information will remain confidential and that consent can be withdrawn at any time, without interference with their usual care.

To minimise and manage any distress or anxiety they may experience completing assessments, the expertise of the principal investigators and follow-up care calls will be employed to identify potential critical situations and provide guidance or referral to further care.

\section{Dissemination}

Findings from this study will be disseminated through peer-reviewed publications and conference presentations to communicate results and clinical implications to health professionals and health policy makers. Presentations on research findings at local community forums will also occur.

\section{DISCUSSION}

This study intends to raise awareness about the risk of psychological distress and poor functional recovery following minor-to-moderate traffic injuries, and to develop processes for the early detection of at-risk individuals, the aim being to prevent disability in the long term.

This study, for the first time, will use biomarkers of autonomic balance, to detect this risk. If results of this study are positive, a non-invasive screening tool like HRV holds promise for providing a means of preventing serious mental health disorders and disability through detection and early treatment, as well as clarifying links between mental and physical health consequences post MVC.

It is expected that general and acute care clinicians would have a crucial role in early diagnosis and referral of at-risk individuals. Employing easy-to-measure 'biological markers' for those at risk of mental health disorders may facilitate timely access to care and help reduce patient-related barriers to mental healthcare. ${ }^{66}$

Adopting the ICF framework to assess consequences of minor-to-moderate injuries also represents a real shift in this field. For example, compared with severe injuries, recovery from these injuries is rarely accepted as a complex multidimensional experience and management often follows a biomedical care model. By contrast, the comprehensive study assessment using standardised measured will clarify the complex needs of people sustaining minor-to-moderate traffic injuries, assist compensation processes and policy changes towards a more holistic approach to injury recovery, and promote comparability with other research.

\section{Author affiliations}

${ }^{1}$ Sydney Medical School—Northern, John Walsh Centre for Rehabilitation Research, University of Sydney, St Leonards, New South Wales, Australia

${ }^{2}$ Northern Clinical School, University of Sydney, St Leonards, New South Wales, Australia

${ }^{3}$ Centre for Vision Research, University of Sydney, Sydney, New South Wales, Australia

${ }^{4}$ Emergency Department, Royal Prince Alfred Hospital, Camperdown, New South Wales, Australia

${ }^{5}$ Emergency Department, Royal North Shore Hospital, St Leonards, New South Wales, Australia

Acknowledgements The authors would like to acknowledge the support of Emergency Department staff at each of the study sites.

Contributors All authors provided inputs in study design. IP, YT and AC are involved in data analysis and data acquisition. IP, AC, BG and IC are responsible for publication writing. All authors reviewed and approved the final version of this manuscript.

Funding This study has received research grant support by the NSW State Insurance Regulatory Authority (grant: MAA 14/1433) and the Australian Rotary Health (lan Scott Mental Health PhD Scholarship).

Competing interests None declared.

Patient consent for publication Not required.

Ethics approval Ethical approval was obtained from the Sydney Local Health District Ethics, Australia (HREC/15/RPAH/423), and Site-Specific approvals obtained for each site (SSA/16/HAWKE/505; RESP/16/349). The study has been prospectively registered with the Australia New Zealand Clinical trial registry on 17 October 2016 (Registration number: ACTRN 12616001445460, IMPRINT study).

Provenance and peer review Not commissioned; externally peer reviewed.

Open access This is an open access article distributed in accordance with the Creative Commons Attribution Non Commercial (CC BY-NC 4.0) license, which permits others to distribute, remix, adapt, build upon this work non-commercially, and license their derivative works on different terms, provided the original work is properly cited, appropriate credit is given, any changes made indicated, and the use is non-commercial. See: http://creativecommons.org/licenses/by-nc/4.0/.

\section{REFERENCES}

1. World Health Organisation. Global status report on road safety 2015. Geneva, Switzerland: WHO, 2015.

2. Alghnam S, Palta M, L Remington P, et al. The association between motor vehicle injuries and health-related quality of life: a longitudinal study of a population-based sample in the United States. Qual Life Res 2014;23:119-27.

3. Polinder S, Haagsma J, Bos N, et al. Burden of road traffic injuries: Disability-adjusted life years in relation to hospitalization 
and the maximum abbreviated injury scale. Accid Anal Prev 2015;80:193-200.

4. Nhac-Vu HT, Hours M, Chossegros L, et al. Prognosis of outcome in adult survivors of road accidents in France: one-year follow-Up in the ESPARR cohort. Traffic Inj Prev 2014;15:138-47.

5. Gabbe BJ, Lyons RA, Fitzgerald MC, et al. Reduced population burden of road transport-related major trauma after introduction of an inclusive trauma system. Ann Surg 2015;261:565-72.

6. Gopinath B, Jagnoor J, Elbers N, et al. Overview of findings from a 2-year study of claimants who had sustained a mild or moderate injury in a road traffic crash: prospective study. BMC Res Notes 2017;10:76

7. Haagsma J, van Beeck E, Toet H, et al. Posttraumatic Stress Disorder Following Injury: Trajectories and Impact on Health-Related Quality of Life. J Depress Anxiety 2013;14:1242-9.

8. Murray CJ, Vos T, Lozano R, et al. Disability-adjusted life years (DALYs) for 291 diseases and injuries in 21 regions, 1990-2010: a systematic analysis for the Global Burden of Disease Study 2010. Lancet 2012;380:2197-223.

9. Papadakaki M, Stamouli M-A, Ferraro OE, et al. Hospitalization costs and estimates of direct and indirect economic losses due to injury sustained in road traffic crashes: results from a one-year cohort study in three European countries (The REHABILAID project). Trauma 2017;19:264-76.

10. Connelly LB, Supangan R. The economic costs of road traffic crashes: Australia, states and territories. Accid Anal Prev 2006;38:1087-93.

11. Guest R, Tran Y, Gopinath B, et al. Psychological distress following a motor vehicle crash: evidence from a statewide retrospective study examining settlement times and costs of compensation claims. BMJ Open 2017;7:e017515.

12. Papadakaki M, Ferraro OE, Orsi C, et al. Psychological distress and physical disability in patients sustaining severe injuries in road traffic crashes: Results from a one-year cohort study from three European countries. Injury 2017;48:297-306.

13. Smith B, Mackenzie-Ross S, Scragg P. Prevalence of poor psychological morbidity following a minor road traffic accident (RTA): The clinical implications of a prospective longitudinal study. Couns Psychol Q 2007;20:149-55.

14. Kenardy J, Edmed SL, Shourie S, et al. Changing patterns in the prevalence of posttraumatic stress disorder, major depressive episode and generalized anxiety disorder over 24 months following a road traffic crash: Results from the UQ SuPPORT study. J Affect Disord 2018;236:172-9.

15. Mayou R, Bryant B. Outcome 3 years after a road traffic accident. Psychol Med 2002;32:671-5.

16. Blanchard EB, Hickling EJ, Taylor AE, et al. Psychiatric morbidity associated with motor vehicle accidents. J Nerv Ment Dis 1995;183:495-504.

17. Kenardy J, Heron-Delaney M, Warren J, et al. Effect of mental health on long-term disability after a road traffic crash: results from the UQ SuPPORT study. Arch Phys Med Rehabil 2015;96:410-7.

18. Jacoby SF, Shults J, Richmond TS. The effect of early psychological symptom severity on long-term functional recovery: A secondary analysis of data from a cohort study of minor injury patients. Int $J$ Nurs Stud 2017;65:54-61.

19. Craig A, Tran Y, Guest R, et al. Psychological impact of injuries sustained in motor vehicle crashes: systematic review and metaanalysis. BMJ Open 2016;6:e011993.

20. Australian Bureau of Statistics. Australia National Survey of Mental Health and Wellbeing 2016. Canberra, Australia: ABS, 2016.

21. World Health Organization. Promoting mental health: Concepts, emerging evidence, practice: Summary report. Geneva, Switzerland: WHO, 2004.

22. Gopinath B, Jagnoor J, Harris IA, et al. Prognostic indicators of social outcomes in persons who sustained an injury in a road traffic crash. Injury 2015;46:909-17.

23. Grant DM, Beck JG, Marques L, et al. The structure of distress following trauma: posttraumatic stress disorder, major depressive disorder, and generalized anxiety disorder. J Abnorm Psychol 2008;117:662-72.

24. Walker FR, Pfingst K, Carnevali L, et al. In the search for integrative biomarker of resilience to psychological stress. Neurosci Biobehav Rev 2017;74:310-20.

25. Duckworth MP, lezzi T. Physical injuries, pain, and psychological trauma: pathways to disability. Psychol Inj Law 2010;3:241-53.

26. Coronas R, Gallardo O, Moreno MJ, et al. Heart rate measured in the acute aftermath of trauma can predict post-traumatic stress disorder: a prospective study in motor vehicle accident survivors. Eur Psychiatry 2011;26:508-12.
27. Kuhn E, Blanchard EB, Fuse T, et al. Heart rate of motor vehicle accident survivors in the emergency department, peritraumatic psychological reactions, ASD, and PTSD severity: a 6-month prospective study. J Trauma Stress 2006;19:735-40.

28. Blanchard EB, Hickling EJ, Galovski T, et al. Emergency room vital signs and PTSD in a treatment seeking sample of motor vehicle accident survivors. J Trauma Stress 2002;15:199-204.

29. Veazey $\mathrm{CH}$, Blanchard EB, Hickling EJ, et al. Physiological responsiveness of motor vehicle accident survivors with chronic posttraumatic stress disorder. Appl Psychophysiol Biofeedback 2004;29:51-62

30. Bryant RA, Harvey AG, Guthrie RM, et al. Acute psychophysiological arousal and posttraumatic stress disorder: a two-year prospective study. J Trauma Stress 2003;16:439-43.

31. Zatzick DF, Russo J, Pitman RK, et al. Reevaluating the association between emergency department heart rate and the development of posttraumatic stress disorder: A public health approach. Biol Psychiatry 2005;57:91-5.

32. Shaikh al arab A, Guédon-Moreau L, Ducrocq F, et al. Temporal analysis of heart rate variability as a predictor of post traumatic stress disorder in road traffic accidents survivors. J Psychiatr Res 2012;46:790-6.

33. Berna G, Ott L, Nandrino JL. Effects of emotion regulation difficulties on the tonic and phasic cardiac autonomic response. PLoS One 2014:9:e102971.

34. Laborde S, Mosley E, Mertgen A. Vagal tank theory: the three rs of cardiac vagal control functioning-resting, reactivity, and recovery. Front Neurosci 2018;12:458.

35. Prinsloo GE, Rauch HG, Derman WE. A brief review and clinical application of heart rate variability biofeedback in sports, exercise, and rehabilitation medicine. Phys Sportsmed 2014;42:88-99.

36. Brosschot JF, Gerin W, Thayer JF. The perseverative cognition hypothesis: a review of worry, prolonged stress-related physiological activation, and health. J Psychosom Res 2006;60:113-24.

37. Porges SW. The polyvagal theory: new insights into adaptive reactions of the autonomic nervous system. Cleve Clin J Med 2009;76(Suppl 2)S86-S90.

38. Thayer JF, Hansen AL, Saus-Rose E, et al. Heart rate variability, prefrontal neural function, and cognitive performance: the neurovisceral integration perspective on self-regulation, adaptation, and health. Ann Behav Med 2009;37:141-53.

39. McCraty R, Shaffer F. Heart rate variability: new perspectives on physiological mechanisms, assessment of self-regulatory capacity, and health risk. Glob Adv Health Med 2015;4:46-61.

40. Balzarotti S, Biassoni F, Colombo B, et al. Cardiac vagal control as a marker of emotion regulation in healthy adults: a review. Biol Psychol 2017;130:54-66.

41. Kok BE, Fredrickson BL. Upward spirals of the heart: autonomic flexibility, as indexed by vagal tone, reciprocally and prospectively predicts positive emotions and social connectedness. Biol Psychol 2010;85:432-6.

42. Lu WC, Tzeng NS, Kao YC, et al. Correlation between health-related quality of life in the physical domain and heart rate variability in asymptomatic adults. Health Qual Life Outcomes 2016;14:149.

43. Beaumont A, Burton AR, Lemon J, et al. Reduced cardiac vagal modulation impacts on cognitive performance in chronic fatigue syndrome. PLoS One 2012;7:e49518.

44. Carnevali L, Koenig J, Sgoifo A, et al. Autonomic and Brain Morphological Predictors of Stress Resilience. Front Neurosci 2018;12:228.

45. Giummarra MJ, Casey SL, Devlin A, et al. Co-occurrence of posttraumatic stress symptoms, pain, and disability 12 months after traumatic injury. Pain Rep 2017;2:e622.

46. Kemp AH, Quintana DS. The relationship between mental and physical health: insights from the study of heart rate variability. Int $J$ Psychophysiol 2013;89:288-96.

47. Sharp TJ, Harvey AG. Chronic pain and posttraumatic stress disorder: mutual maintenance? Clin Psychol Rev 2001;21:857-77.

48. McLean SA, Clauw DJ, Abelson JL, et al. The development of persistent pain and psychological morbidity after motor vehicle collision: integrating the potential role of stress response systems into a biopsychosocial model. Psychosom Med 2005;67:783-90.

49. Lahera G, Gálvez JL, Sánchez P, et al. Functional recovery in patients with schizophrenia: recommendations from a panel of experts. BMC Psychiatry 2018;18:176.

50. World Health Organization. International Classification of Functioning, Disability and Health: ICF. Geneva, Switzerland: WHO, 2001.

51. Herbert RD. Cohort studies of aetiology and prognosis: they're different. J Physiother 2014;60:241-4.

52. Greenspan L, McLellan BA, Greig H. Abbreviated Injury Scale and Injury Severity Score: a scoring chart. J Trauma 1985;25:60-4. 
53. New South Wales Institute of Trauma and Injury Management. The NSW Trauma Registry profile of serious to critical injuries 2009. Sydney: NSW Health, 2012.

54. Curtis KA, Mitchell RJ, Chong SS, et al. Injury trends and mortality in adult patients with major trauma in New South Wales. Med J Aust 2012;197:233-7

55. Cameron PA, Gabbe BJ, Cooper DJ, et al. A statewide system of trauma care in Victoria: effect on patient survival. Med J Aust 2008;189:546-50.

56. Jagnoor J, Blyth F, Gabbe B, et al. Factors influencing social and health outcomes after motor vehicle crash injury: an inception cohort study protocol. BMC Public Health 2014:14:199.

57. Faul F. G* Power Version 3.1. 9.2. Germany, Kiel: Universitat Kiel, 2014.

58. Henry JD, Crawford JR. The short-form version of the Depression Anxiety Stress Scales (DASS-21): construct validity and normative data in a large non-clinical sample. Br J Clin Psychol 2005;44:227-39.

59. Weiss DS, Marmar CR. The Impact of Event Scale-Revised. In: Wilson JP, Keane TM, eds. Assessing psychological trauma and PTSD: a practitioner's handbook. Guilford, 1997:399-411.

60. Diagnostic and statistical manual of mental disorders (DSM-5®). Arlington: American Psychiatric Association, 2013.

61. Laborde S, Mosley E, Thayer JF. Heart rate variability and cardiac vagal tone in psychophysiological research-recommendations for experiment planning, data analysis, and data reporting. Front Psychol 2017;8:213.

62. Tran $\mathrm{Y}$, Wijesuriya N, Tarvainen $\mathrm{M}$, et al. The relationship between spectral changes in heart rate variability and fatigue. J Psychophysio 2009;23:143-51.

63. Stroop JR. Studies of interference in serial verbal reactions. J Exp Psychol 1992;121:15-23.

64. Bradley MM, Lang PJ. Measuring emotion: the self-assessment manikin and the semantic differential. J Behav Ther Exp Psychiatry 1994;25:49-59.
65. Tarvainen MP, Niskanen JP, Lipponen JA, et al. Kubios HRV-heart rate variability analysis software. Comput Methods Programs Biomed 2014;113:210-20.

66. Craig A, Tran Y, Craig M. Stereotypes towards stuttering for those who have never had direct contact with people who stutter: a randomized and stratified study. Percept Mot Skills 2003;97:235-45

67. Crawford JR, Henry JD. The positive and negative affect schedule (PANAS): construct validity, measurement properties and normative data in a large non-clinical sample. $\mathrm{Br} J$ Clin Psychol 2004;43:245-65.

68. World Health Organisation. World Health Organization disabilty assessment schedule: WHODAS II. Phase 2 field trials. Health services research. Geneva, Switzerland: WHO, 2000.

69. Williamson A, Hoggart B. Pain: a review of three commonly used pain rating scales. J Clin Nurs 2005;14:798-804.

70. Linton SJ, Nicholas M, MacDonald S. Development of a short form of the Örebro Musculoskeletal Pain Screening Questionnaire. Spine 2011;36:1891-5.

71. Wewers ME, Lowe NK. A critical review of visual analogue scales in the measurement of clinical phenomena. Res Nurs Health 1990;13:227-36

72. Teasdale G, Jennett B. Assessment of coma and impaired consciousness. a practical scale. Lancet 1974;2:81-4.

73. Ware JE, Kosinski M, Turner-Bowker DM, et al. How to Score Version 2 of the SF-12 Health Survey (with a Supplement Documenting Version 1). In: Lincoln RI, ed. QualityMetric Inc.; Health Assessment Lab. Boston, MA, USA, 2005.

74. Carver CS, Scheier MF, Weintraub JK. Assessing coping strategies: a theoretically based approach. J Pers Soc Psychol 1989;56:267-83.

75. Sullivan MJL, Bishop SR, Pivik J. The pain catastrophizing scale: development and validation. Psychol Assess 1995;7:524-32.

76. Luszczynska A, Scholz U, Schwarzer R. The general self-efficacy scale: multicultural validation studies. J Psychol 2005;139:439-57. 